\title{
Utilizing the precessing orbit of TRMM to produce hourly corrections of geostationary infrared imager data with the VIRS sensor Benjamin Scarino*a, David R. Doelling ${ }^{\mathrm{b}}$, Conor Haney ${ }^{\mathrm{a}}$, Kristopher Bedka ${ }^{\mathrm{b}}$, Patrick Minnis ${ }^{\mathrm{b}}$, Arun Gopalan ${ }^{\mathrm{a}}$, Rajendra Bhatt ${ }^{\mathrm{a}}$ \\ ${ }^{a}$ SSAI, One Enterprise Pkwy Ste 200, Hampton, VA 23666 USA \\ ${ }^{\mathrm{b}}$ NASA Langley Research Center, 21 Langley Blvd MS 420, Hampton, VA 23681-2199 USA
}

\begin{abstract}
Accurate characterization of the Earth's radiant energy is critical for many climate monitoring and weather forecasting applications. For example, groups at the NASA Langley Research Center rely on stable visible- and infraredchannel calibrations in order to understand the temporal/spatial distribution of hazardous storms, as determined from an automated overshooting convective top detection algorithm. Therefore, in order to facilitate reliable, climate-quality retrievals, it is important that consistent calibration coefficients across satellite platforms are made available to the remote sensing community, and that calibration anomalies are recognized and mitigated. One such anomaly is the infrared imager brightness temperature (BT) drift that occurs for some Geostationary Earth Orbit satellite (GEOsat) instruments near local midnight. Currently the Global Space-Based Inter-Calibration System (GSICS) community uses the hyperspectral Infrared Atmospheric Sounding Interferometer (IASI) sensor as a common reference to uniformly calibrate GEOsat IR imagers. However, the combination of IASI, which has a 21:30 local equator crossing time (LECT), and hyperspectral Atmospheric Infrared Sounder (AIRS; 01:30 LECT) observations are unable to completely resolve the GEOsat midnight BT bias. The precessing orbit of the Tropical Rainfall Measuring Mission (TRMM) Visible and Infrared Scanner (VIRS), however, allows sampling of all local hours every 46 days. Thus, VIRS has the capability to quantify the BT midnight effect observed in concurrent GEOsat imagers. First, the VIRS IR measurements are evaluated for long-term temporal stability between 2002 and 2012 by inter-calibrating with Aqua-MODIS. Second, the VIRS IR measurements are assessed for diurnal stability by inter-calibrating with Meteosat-9 (Met-9), a spin-stabilized GEOsat imager that does not manifest any diurnal dependency. In this case, the Met-9 IR imager is first adjusted with the official GSICS calibration coefficients. Then VIRS is used as a diurnal calibration reference transfer to produce hourly corrections of GEOsat IR imager BT. For the 9 three-axis stabilized GEO imagers concurrent with VIRS, the midnight effect increased the BT on average by $0.5 \mathrm{~K}(11 \mu \mathrm{m})$ and $0.4 \mathrm{~K}(12 \mu \mathrm{m})$, with a peak at 01:00 local time. As expected, the spin-stabilized GEOsats revealed a smaller diurnal temperature cycle (mostly $<0.2 \mathrm{~K}$ ) with inconsistent peak hours.
\end{abstract}

\section{INTRODUCTION}

Consistent, well-calibrated radiances are fundamental to the development of long-term satellite-based climate data records (CDRs). A variety of satellite-borne imaging radiometers have similar but spectrally unequal channels that differ in response characteristics and calibration. Data from any properly calibrated satellite imager could contribute to the development of long-term CDRs. For example, NASA LaRC is processing more than 20 years of the Geostationary Operational Environmental Satellite system (GOES) infrared (IR) and visible data record with an automated hazardous storm detection algorithm in order to understand the temporal/spatial distribution of these storms and how they have changed over time (Fig. 1). In this case, the storm frequency is analyzed diurnally ${ }^{1}$. Furthermore, the NASA Clouds and the Earth's Radiant Energy System (CERES) project has, since 2000, utilized the five contiguous Geostationary Earth Orbit satellite (GEOsat) imagers in order to estimate the regional diurnal shortwave (SW) and longwave (LW) broadband fluxes and cloud properties to supplement Terra and Aqua CERES measurements ${ }^{2,3}$. Therefore, intra-series calibration consistency is critical for minimizing sensor-induced trends, as well as for mitigating any imager-sourced diurnal biases. There are a variety of independent and inter-consistent traceability standards for visible channels given that many GEOsat imagers do or did not maintain a reliable onboard calibration system, such as a solar diffuser ${ }^{3,4}$. For IR channels, however, most operational meteorological satellites have relied on onboard calibration systems for their IR channels, which have ensured stability over the operational records.

Keywords: geostationary, GOES, TRMM, VIRS, Meteosat, IASI, GSICS, infrared, vicarious calibration *benjamin.r.scarino@nasa.gov; $\quad$ phone1757951-1622; fax 1757 951-1902 


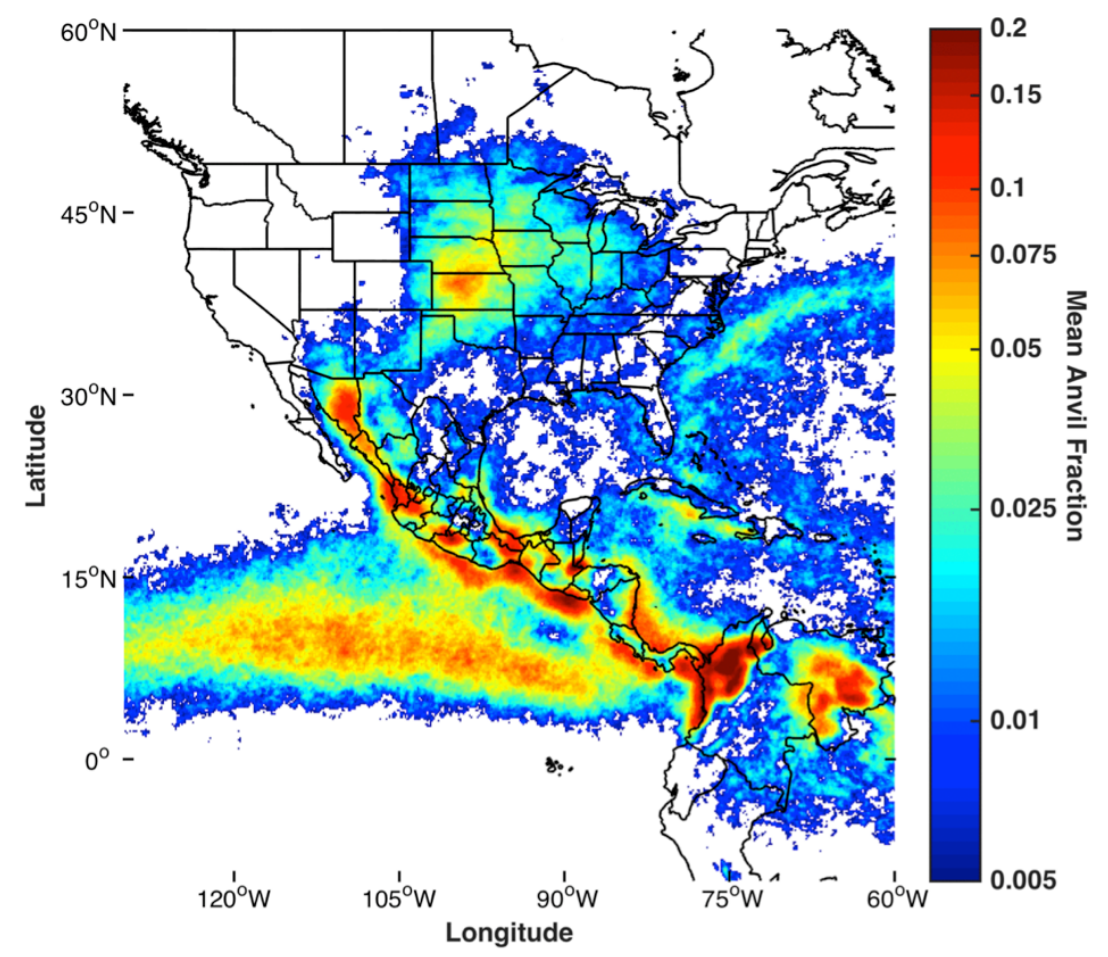

Figure 1. July gridded anvil cloud fraction from 1996 through 2015 at 00:00 local time.

The World Meteorological Organization Global Space-based Inter-Calibration System (GSICS) was developed to satisfy the need for consistent, absolute-reference calibration coefficients across space-based imagers ${ }^{5}$. The GSICS community has sought to correct calibration inconsistencies among modern GEOsats and Low Earth Orbit operational satellites. The GSICS effort utilizes either Infrared Atmospheric Sounding Interferometer (IASI) or Atmospheric Infrared Sounder (AIRS) hyperspectral radiances as the reference for calibrating GEOsat imager infrared channels ${ }^{6}$, the calibration products of which are currently online (http://gsics.wmo.int/). The chief goal among the participating satellite agencies within GSICS is to make the international suite of satellites consistent through common practices and reference standards. However, the MetOp-A (launched in 2006) IASI has a 21:30 local equator crossing time (LECT) and Aqua (launched in 2003) AIRS (01:30 LECT) hyperspectral measurements cannot completely resolve diurnal GEOsat brightness temperature (BT) biases.

Many IR imagers onboard three-axis-stabilized GEOsat platforms are influenced by anomalous IR-imager drift during the hours near local midnight. This anomaly has been extensively documented for the GOES series of satellites, and is thought to be caused by solar heating of the instrument, which is heightened near local midnight. The calibration issue, known as the midnight calibration anomaly or the midnight effect, manifests as a change in the magnitude of instrument-derived calibration gains for GEOsat imager IR channels during the approximately eight hours centered on satellite midnight ${ }^{7,8,9}$. For the GOES-8 through GOES-14 series of satellites, the midnight anomaly has been well documented, along with a means for mitigation ${ }^{7,89}$. However, NOAA has acknowledged that the midnight anomaly effects GOES-15, the Multifunctional Transport Satellite (MTSAT), and the Communication, Ocean, and Meteorological Satellite (COMS) as well ${ }^{10,11}$.

The midnight effect is most pronounced in the shortwave IR (SWIR; $3.9 \mu \mathrm{m})$ and water vapor $(6.7 \mu \mathrm{m})$ channels, but is also apparent in the longwave (LW) channels $(11,12 \text {, and } 13 \mu \mathrm{m})^{8,12}$. The calibration instability is driven by thermal stress, in that radiation from excessively heated surfaces in the imager's scan cavity is reflected by the blackbody reference to the detector during the blackbody calibration sequence, which is performed every 30 minutes ${ }^{9,10}$. The result is a temporary decrease in the magnitude of the calibration gain, which causes the Earth to appear cooler by 0.6 to $1.0 \mathrm{~K}$, for LW and SWIR channels, repectively ${ }^{7}$. Instruments onboard spin-stabilized satellites do not appear to be influenced by a midnight-effect-induced diurnal calibration instability ${ }^{12}$. Note that another type of anomaly, which 
contaminates the space-look calibration sequence, occurs during the two annual eclipse seasons, but this manuscript does not address that effect ${ }^{\top}$.

A midnight blackbody calibration correction (MBCC) was implemented by NOAA for GOES imagers to account for the systematic gain drift, which is based on the observation that when the midnight effect is absent, there exists high correlation between the gain and the temperature of several optical components, and thus bad gain values can be replaced by estimates computed by the correlation regression during the optimal period. That is, the operational correction is is based on the assumption that the daytime gains are truth, as the gain is related to a sample of optics temperatures that exclude data from the eight hours surrounding midnight ${ }^{7,9}$. In practice, the MBCC has been shown to significantly improve midnight calibration accuracy for shortwave and 12-/13- $\mu \mathrm{m}$ GOES channels ${ }^{13,14,15}$. For $11-\mu \mathrm{m}$ GOES channels, however, implementation of MBCC can still leave about a $0.5-\mathrm{K}$ difference relative to the daytime reference data unaccounted for ${ }^{12}$. Tests of MBCC applied for MTSAT-2 revealed similar results as those for GOES imagers $^{12}$. Therefore, given that the MBCC only partially alleviates the midnight effect, NOAA and the Korea Meteorological Administration (KMA) are collaborating to deliver an improved means of correcting for extra radiation viewed by the detector, and thus improve the midnight accuracy for the GOES, MTSAT, and COMS satellite series, thereby reducing risk for future advanced imagers ${ }^{10,11}$. Results of MBCC implementation for the Spinning Enhanced Visible and Infrared Imager (SEVIRI) IR channels of spin-stabilized Meteosat-9 (Met-9) expectedly revealed stable diurnal consistency relative to both AIRS and IASI with biases less than $0.2 \mathrm{~K}$. The result thereby supports the idea that imagers mounted onboard spin-stabilized platforms are not susceptible to the heat-stress cause of the midnight effect, whereas those on three-axis-stabilized platforms are ${ }^{12}$.

The GEOsat BT midnight effect can be demonstrated by the slope discrepancies between daytime and nighttime ray-matched BT pairs involving either GOES-East or GOES-West with Terra- or Aqua-MODIS. Figures 2a (Aqua) and 2c (Terra) highlight the daytime and nighttime slope difference for GOES-10 (West). If there was no GOES-10 diurnal dependency, the Terra (10:30 LECT) or Aqua (13:30 LECT) daytime and nighttime slopes should be equal. However, the current GSICS approach does not account for the midnight effect given than the IASI inter-calibration corrections are only applicable to MetOp-A overpass times of 09:30 LECT. Similarly, the MODIS and AIRS comparisons are reasonably only valid during the Aqua overpass times. Therefore, the constellation of IASI, AIRS, and MODIS sensors does not encompass the entire diurnal cycle. It is encouraging to see that the GEOsat blackbody does maintain stable BT when compared with MODIS. However, there are gain discontinuities between GOES satellite records, indicating that the absolute value of the GEOsat BT varies between imagers with respect to MODIS. Employing the GSICS IASI/GEOsat IR inter-calibration algorithm should place all GEOsat IR records on the same radiometric scale during IASI overpass times, but this technique does not account for the diurnal bias.

The Tropical Rainfall Measuring Mission (TRMM) satellite maintains a precessing orbit between $\pm 30^{\circ}$ latitude, which allows it to acquire Visible and Infrared Scanner (VIRS) imager measurements covering all tropical regions for all local hours every 46 days. The GEOsat and VIRS ray-matching opportunities can achieve a similar hourly viewing angle distribution, whereas for sun-synchronous satellites, the ray-matched viewing angle distribution is dependent on the local overpass hour. That is, nadir ray-matching events can only be achieved during a sun-synchronous satellite's orbit LECT. For increased time after the orbit LECT, the viewing conditions become more disparate. It it has been reported that the GOES imager scan mirror may have a slight angular dependency ${ }^{12}$. Such a dependency might influence sun-synchronous inter-calibration events for ray-matches not centered on the LECT. This GEOsat dependence is not a problem for VIRS inter-calibrations because the TRMM precessing orbit allows VIRS to sample near the GEOsat sub-satellite point for all local hours, thereby acquiring an even distribution of viewing angle matches across the domain.

The goal of this work is to quantify both the magnitude and the peak of the GEOsat BT midnight effect utilizing VIRS and GEOsat hourly inter-calibrations. First, the VIRS IR sensor long-term stability is validated and possibly corrected with respect to Aqua-MODIS. Second the VIRS IR sensor diurnal stability is evaluated and possibly corrected with respect to Met-9, which is a spin-stabilized GEOsat imager. The Met-9 IR BT are first corrected using the GSICSprovided calibration coefficients, which use MetOp-A IASI as a reference. Then the Met-9 GSICS reference IR calibration is transferred to VIRS. The VIRS (GSICS-referenced) BT are then compared directly to IASI measurements to confirm that the VIRS BT have been properly referenced to IASI during the IASI LECT. Finally, the GEOsat IR sensors are calibrated through subsequent hourly ray-matching of the imager measurements with VIRS (GSICS- or IASI-referenced) in order to resolve the magnitude and timing of the GEOsat midnight effect. The midnight effect should be more consistent for three-axis-stabilized, rather than for spin-stabilized, GEOsats. 

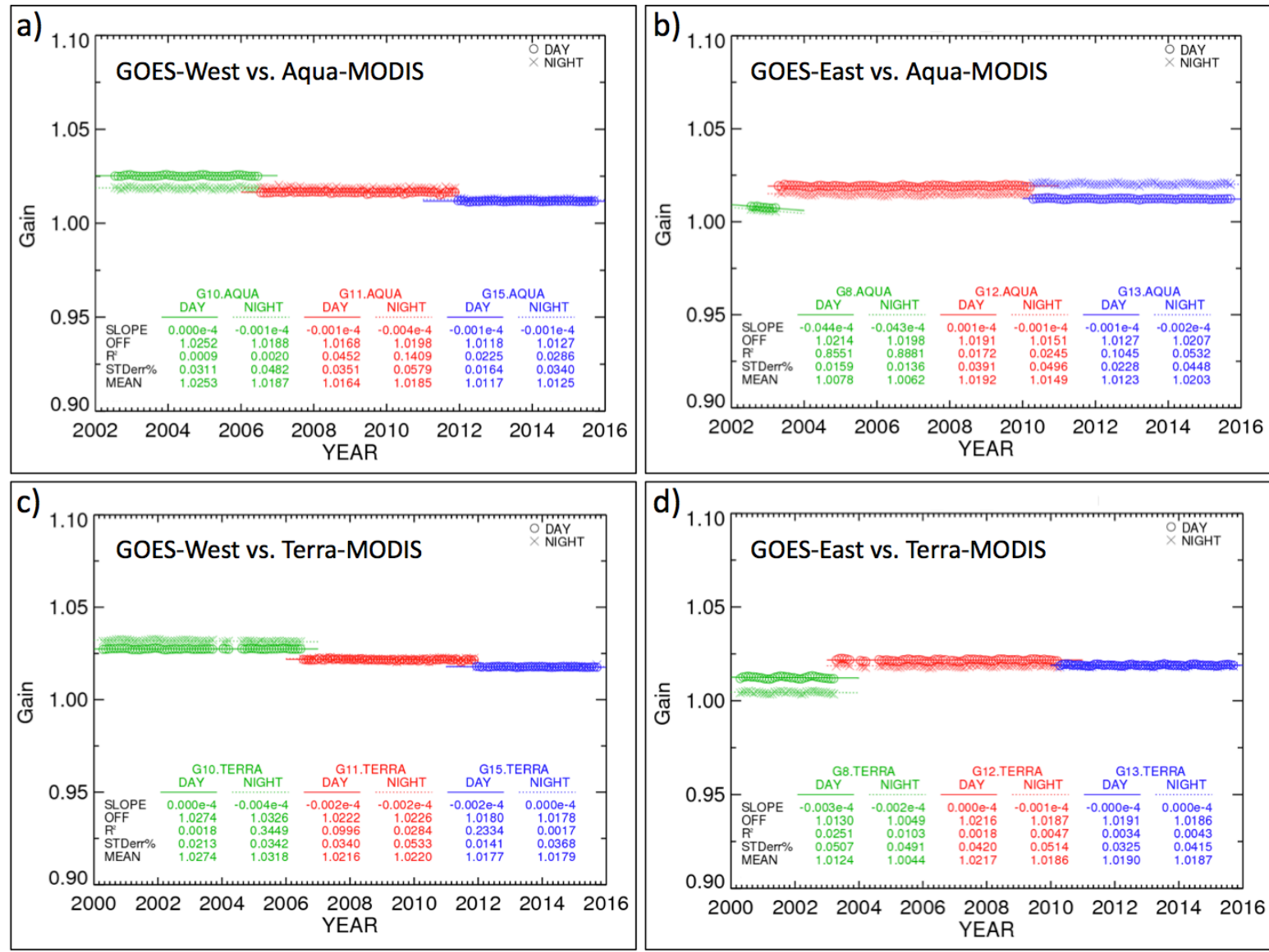

Figure 2. Aqua-MODIS (top row; 13:30 LECT) and Terra-MODIS (bottom row; 10:30 LECT) day and night calibration gain trends for the GOES-West (left column) and GOES-East (right column) satellite series. Note the extremely small trend slopes (year ${ }^{-1}$ ), except for the short GOES-8 record in b), indicating that the GOES blackbodies are stable with respect to MODIS.

\section{DATA AND METHODOLOGY}

Although GEOsat calibration coefficients were produced for 14 and 9 imagers for the, respective, $11-\mu \mathrm{m}$ and $12-\mu \mathrm{m}$ VIRS IR imager channels (see Section 3), the methodology is primarily demonstrated using the GOES-13 $11-\mu \mathrm{m}$ channel (i.e., channel 4). Given the challenges of solar contribution for the 3.8- $\mu \mathrm{m}$ channel, provision of hourly calibration coefficients for that spectral range are left for a follow-on study.

\subsection{VIRS Stability Verification}

Because VIRS will be the means of diurnally calibrating the GEOsats, it is important to first establish the stability of the VIRS IR channels. The 14 GEOsats calibrated in this study cover most of the VIRS record, and VIRS stability is required for consistent calibration across the GEOsat constellation. We have chosen Aqua-MODIS as the independent stability reference given its extremely stable onboard blackbody temperature used for calibrating all mid-wave and longwave IR spectral regions with wavelengths from 3.7 to $14.4 \mu \mathrm{m}^{16}$. Figure 3 reveals simultaneous nadir overpass (SNO) comparisons between Aqua-MODIS and VIRS for the 3.8-, 11-, and 12- $\mu \mathrm{m}$ wavelengths of VIRS - channels 3, 4, and 5 respectively. The SNOs are acquired between $\pm 30^{\circ}$ latitude for orbit intersections within 15 minutes, and for viewing zenith angles less than $5^{\circ}$. The monthly mean BT bias is computed monthly and plotted for daytime (red) and nighttime (blue) ray-matching events from 2002 through 2012. There is a period of missing VIRS data in 2011, but this gap should not significantly influence the continuity of the stability assessment. For all three channels, the VIRS BT is extremely stable relative to MODIS with maximum degradation not in excess of $0.02 \mathrm{~K}$ per decade. Therefore, VIRS is suitable to uniformly calibrate the GEOsats throughout its record. In this assessment, neither MODIS nor VIRS has been referenced 

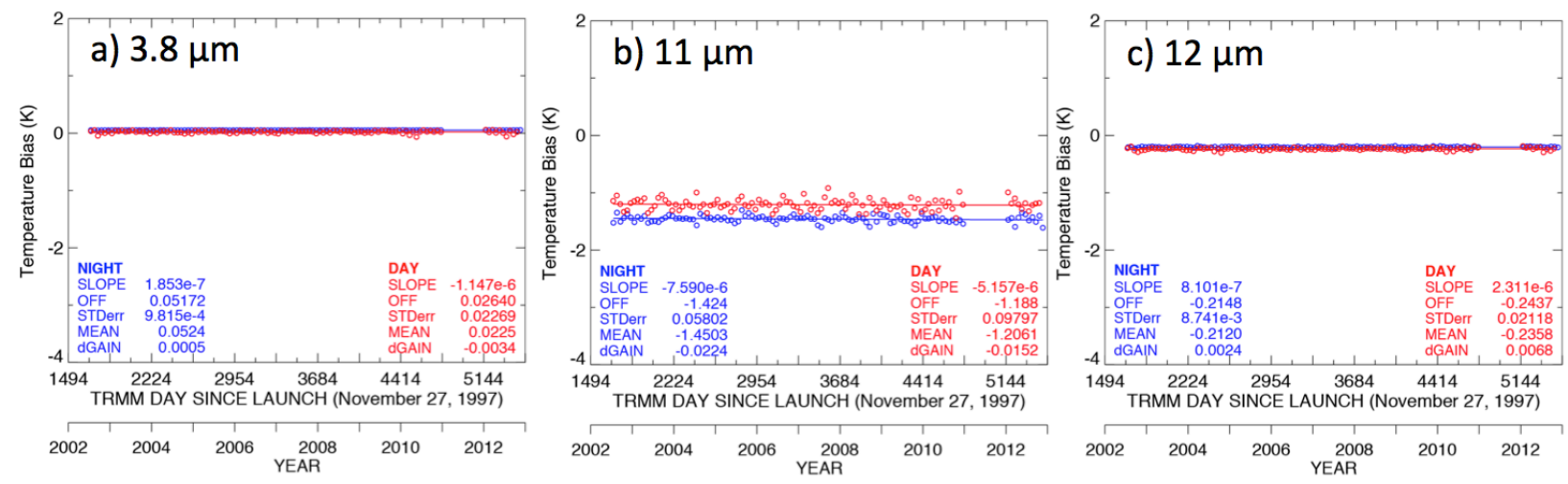

Figure 3. Mean VIRS - MODIS bias trend from 2002 through 2012 for the a) 3.8- $\mu \mathrm{m}, \mathrm{b}) 11-\mu \mathrm{m}$, and c) $12-\mu \mathrm{m}$ channels. Missing VIRS data for 2011 does not influence the near-zero degradation. The linear trend slope and offset are based on days since TRMM launch. The slope units are day ${ }^{-1}$.

to a common calibration reference, such as IASI, and therefore the temperature bias is the sensor-based BT difference. The task of referencing VIRS BT to IASI is described in the next section.

\subsection{VIRS Standardized to Met-9 (GSICS-corrected)}

The VIRS instrument must first be referenced to the IASI GSICS standard for each hour. This task is accomplished by inter-calibrating VIRS with Met-9, which has first been corrected using the GSICS Met-9 calibration coefficients from the GSICS MSG-2 SEVIRI IASI-A Reanalysis Correction product (https://www.star.nesdis.noaa.gov/smcd/GCC/ProductCatalog.php) during 2010. Meteosat-9 is spin-stabilized and therefore assumed to be resistant to midnight heating effects due to there being little opportunity for differential heating across the satellite bus. The VIRS/GEOsat ray-matching methodology uses all $0.5^{\circ}$ by $0.5^{\circ}$ gridded mean BT pairs, that have a viewing angle difference of less than $5^{\circ}$ and are coincident within 15 minutes. The mean BT is computed by averaging the pixel-level radiances and converting the average radiance to BT using the Planck function based on the central wavelength. A $50-\mathrm{km}$ BT standard deviation $\left(\sigma_{\mathrm{BT}}\right)$ is used to mitigate any time-discrepancy-induced cloud displacement, navigation error, or viewing angle difference parallax effects. A linearly sliding threshold for $\sigma_{\mathrm{BT}}$ of $1.5 \%$ at $300 \mathrm{~K}$ and $7.5 \%$ at $200 \mathrm{~K}$ ensures a robust dynamic measurement range by preserving more of the less-frequent cold/cloudy ray-matching events, while increasing the spatial homogeneity threshold for the more frequently sampled near-clear-sky events. The tropics provide the greatest dynamic range, as they contain both rather cold deep convective clouds and warm ocean scenes. The reference Met-9 (GSICS-corrected) BT is converted to an equivalent target (VIRS) BT using the spectral band adjustment coefficients found in Tables 1 and 2 (see Section 2.6). Hourly VIRS and Met-9 $50-\mathrm{km}$ ray-matched BT pairs are linearly regressed monthly. Figure $4 \mathrm{a}$ shows the ray-matched comparison results of VIRS and Met-9 (GSICS-corrected) BT for the 11- $\mu \mathrm{m}$ channel during the 18-19 GMT interval.

The principle components (PC; i.e., orthogonal regression, or perpendicular distance to the line) slope and $\mathrm{x}$-axis offset values indicate a temperature-dependent bias, which is apparent for each hour as illustrated in Fig. 4b. The red and blue arrows of Fig. 4a correspond to the Met-9 (GSICS-corrected) minus VIRS biases at 290 and 220 K, respectively, as correspondently indicated on Fig. 4b at the 18-19 GMT interval. In an effort to mitigate hour-to-hour instability, the resultant statistics for any GMT interval represent the three-hour culmination of ray-matched points centered on that interval. Figure $4 \mathrm{~b}$ reveals that the temperature-dependent bias intensity, especially at $220 \mathrm{~K}$, varies as a function of GMT interval, which may suggest a diurnal dependence in either the VIRS- or the Met-9 (GSICS-corrected) data. The VIRS (observed) BT values are normalized to Met-9 (GSICS-corrected) by applying the VIRS/Met-9 (GSICS-corrected) PC hourly slope and offset corrections, thus yielding GSICS-referenced VIRS BT as follows:

$$
B T_{V I R S(G S I C S-r e f e r e n c e d)}=\operatorname{slope}(G M T \text { interval }) \times\left(B T_{V I R S(\text { observed })}-\text { off } \operatorname{set}(G M T \text { interval })\right) \text {, }
$$

The Met-9 sub-satellite longitude of $0^{\circ} \mathrm{E}$ determines the GMT interval. 

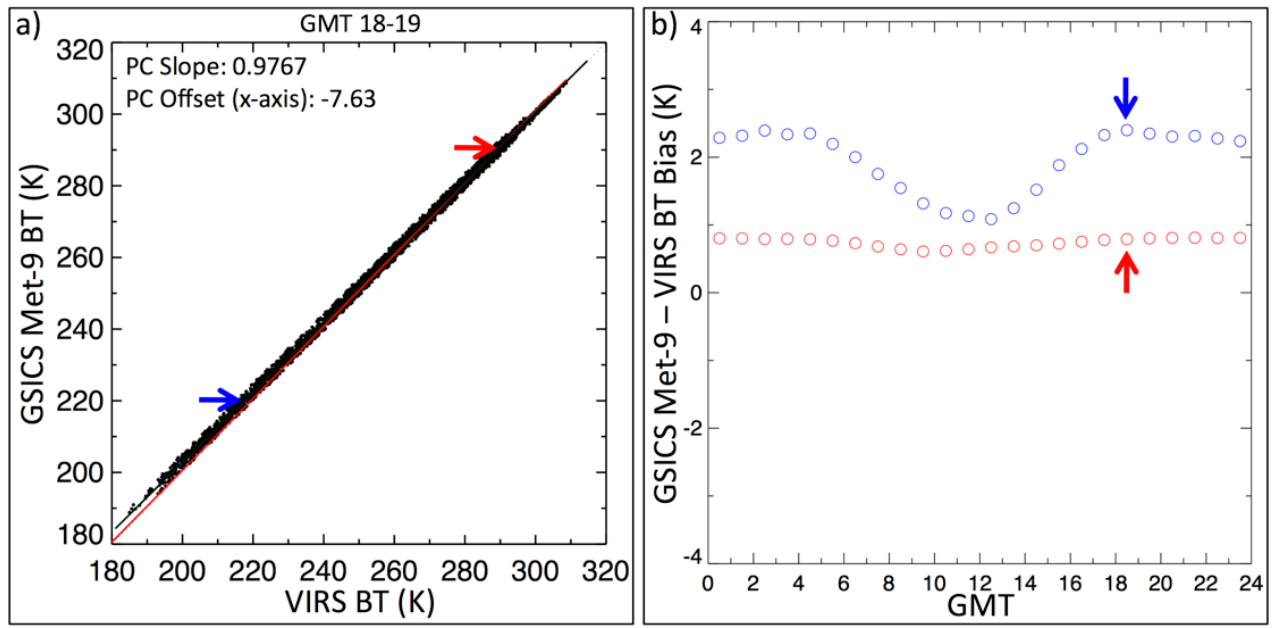

Figure 4. VIRS and Met-9 (GSICS-corrected) 11- $\mu \mathrm{m}$ BT ray-matched bias results for a) 18-19 GMT culminated from January through December 2010, with principle components slope and x-axis offset. The blue and red arrows correspond to temperature-dependent GSICS-consistent Met-9 minus VIRS biases at 290 and $220 \mathrm{~K}$, respectively, as shown on the b) hourly bias plot.

\subsection{VIRS (GSICS-referenced) Compared with IASI}

To verify that the Met-9 and VIRS BT diurnal variation is mostly due to the VIRS sensor, the VIRS (observed) BT values are compared directly with those of IASI. Because Met- 9 is located at $0{ }^{\circ} \mathrm{E}$, the GMT and local hour are the same. The MetOp-A satellite passes the Met-9 GEO domain near 09:30 and 21:30 GMT. For the VIRS and Met-9 (GSICScorrected) comparison (Fig. 4), the 09:30 GMT result occurs during the 220-K BT difference drift, whereas the 21:30 GMT comparison occurs after the drift (Fig. 4b). As such, the VIRS (observed) BT are compared to IASI near these times, the results of which are shown in Fig. 5. The daytime IASI minus VIRS BT differences are +0.5 and $+0.9 \mathrm{~K}$ at 290 and $220 \mathrm{~K}$, respectively (Fig. 5a), whereas the Met-9 (GSICS-corrected) minus VIRS BT differences are +0.6 and $+1.3 \mathrm{~K}$ at 290 and $220 \mathrm{~K}$, respectively (Fig. 4b). For nighttime, the respective 290- and 220-K IASI BT differences are +0.7 and $+1.8 \mathrm{~K}$, and those for Met-9 (GSICS-corrected) are +0.8 and $+2.2 \mathrm{~K}$. The VIRS BT difference with respect to either IASI or Met-9 (GSICS-corrected) varies by 0.1 to $0.4 \mathrm{~K}$, and the diurnal dependency is more than twice as disparate, but comparable between the two sources at both temperature levels. The day and night 290-K BT difference range is at most $0.2 \mathrm{~K}$, e.g., 0.5 vs. $0.7 \mathrm{~K}$ with respect to IASI, and 0.6 vs. $0.8 \mathrm{~K}$ with respect to Met-9 (GSICScorrected). The 220-K BT diurnal VIRS with IASI or Met-9 (GSICS-corrected) difference range is $0.9 \mathrm{~K}$, being either 0.9 or $1.3 \mathrm{~K}$ during the day, to either 1.8 or $2.2 \mathrm{~K}$ at night. This demonstrates that VIRS, rather than Met- 9 , is the source the 220-K diurnal drift (also, because Met-9 is spin-stabilized, it is assumed to be diurnally consistent ${ }^{12}$ ). That is, the diurnal dependency of the VIRS (GSICS-referenced) BT values relative to Met-9 at the IASI overpass times matches that of VIRS (observed) relative to IASI directly, thereby indicating that the VIRS (GSICS-referenced) data have been successfully normalized to the GSICS-IASI standard.

\subsection{GOES-13 Compared with VIRS (observed)}

Another verification that VIRS has a daytime 220-K BT drift is the ray-matched comparison of GOES-13 with VIRS (observed), i.e., before VIRS is referenced to GSICS. The same Met-9/VIRS ray-matching procedure discussed in Section 2.2 is employed here. Figure 6 shows the ray-matched comparison results of GOES-13 and VIRS (observed) for the 11- $\mu \mathrm{m}$ channel, for the 18-19 GMT interval (Fig. 6a), and the BT-dependent bias as a function of GMT interval (Fig. 6b). Owing to data availability, the ray-matching period for this case was split temporally, consisting of collection from May through December 2010 and January through April 2012. The 220-K local noon drift magnitude is at least $1 \mathrm{~K}$ for both GOES-13 (17 GMT) and Met-9 (12 GMT), thus further supporting that this local bias maximum is a VIRS feature. Unlike Fig. 4b, however, Fig. 6b reveals a local-midnight-centered anomaly in the 290-K biases, which, based on comparison with Fig. 4b, is likely sourced from GOES-13 rather than VIRS. The clear presence of the GOES-13 midnight (05 GMT) effect in Fig. 6b illustrates the need for this VIRS-dependent calibration approach. 

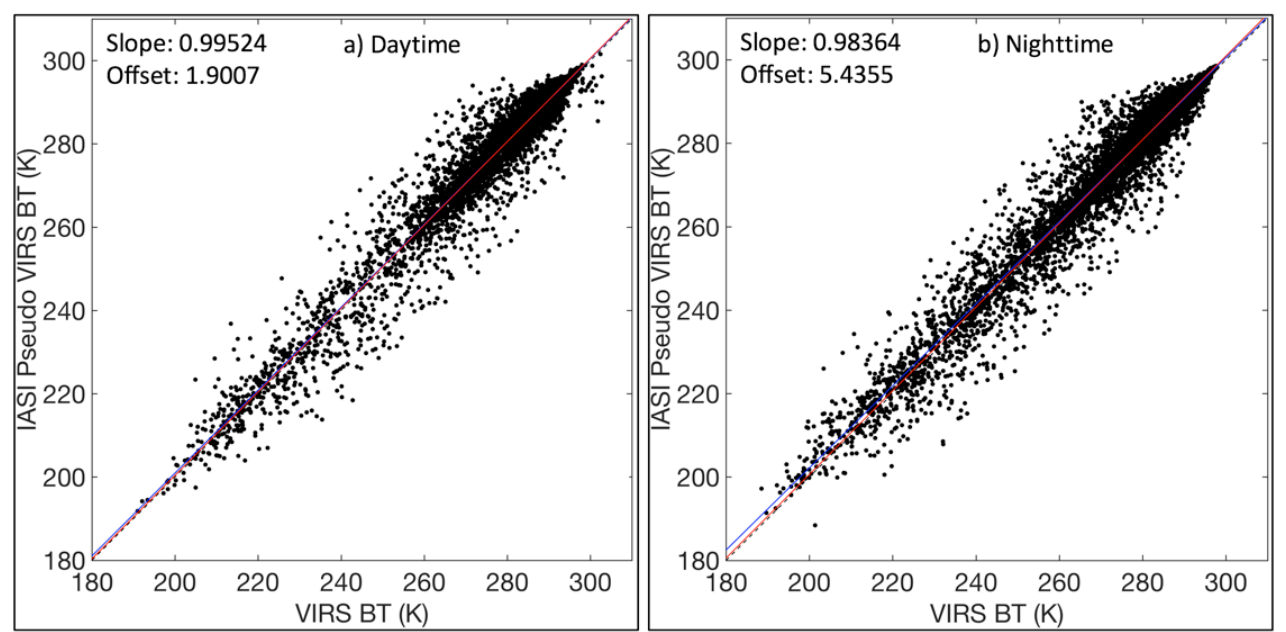

Figure 5. The MetOp-A IASI and TRMM VIRS (observed) ray-matched BT pairs for a) daytime (09:30 GMT) and b) nighttime (21:30 GMT) during June through August 2012.
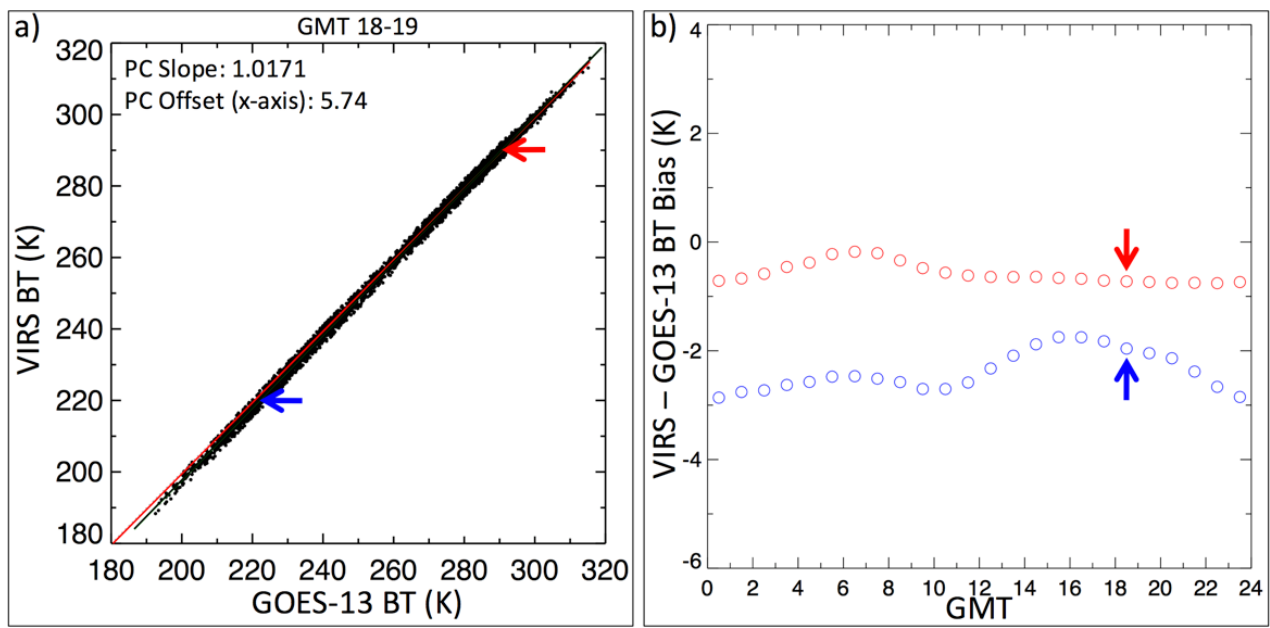

Figure 6. GOES-13 and VIRS (observed) 11- $\mu \mathrm{m}$ BT ray-matched bias results for a) 18-19 GMT culminated from May through December 2010 and January through April 2012 with principle components slope and x-axis offset. The blue and red arrows correspond to temperature-dependent VIRS minus GOES-13 biases at 290 and $220 \mathrm{~K}$ as shown on the b) hourly bias plot.

\subsection{GOES-13 Standardized to VIRS (GSICS referenced)}

The VIRS (GSICS-referenced using Eq. 1) data can now be used to yield GMT-dependent GEOsat correction factors. Similar to Fig. 6, Fig. 7 shows ray-matched comparison results, but between GOES-13 and GSICS-referenced VIRS BT. The BT-dependent bias has decreased as indicated by the diminished PC x-axis offset and slope nearer to 1.0 (Fig. 7a), and by the reduced magnitude of difference between the $290-\mathrm{K}$ and $220-\mathrm{K}$ bias indicators, which is now significantly more consistent across all GMT intervals (Fig. 7b). Also, the VIRS local-noon 220-K BT drift has been mitigated, but the GOES-13 midnight effect drift is still preserved. Put simply, Fig. 7 illustrates the hourly adjustments necessary for obtaining diurnally consistent GOES-13 (GSICS-referenced) $11-\mu \mathrm{m}$ BT values. 

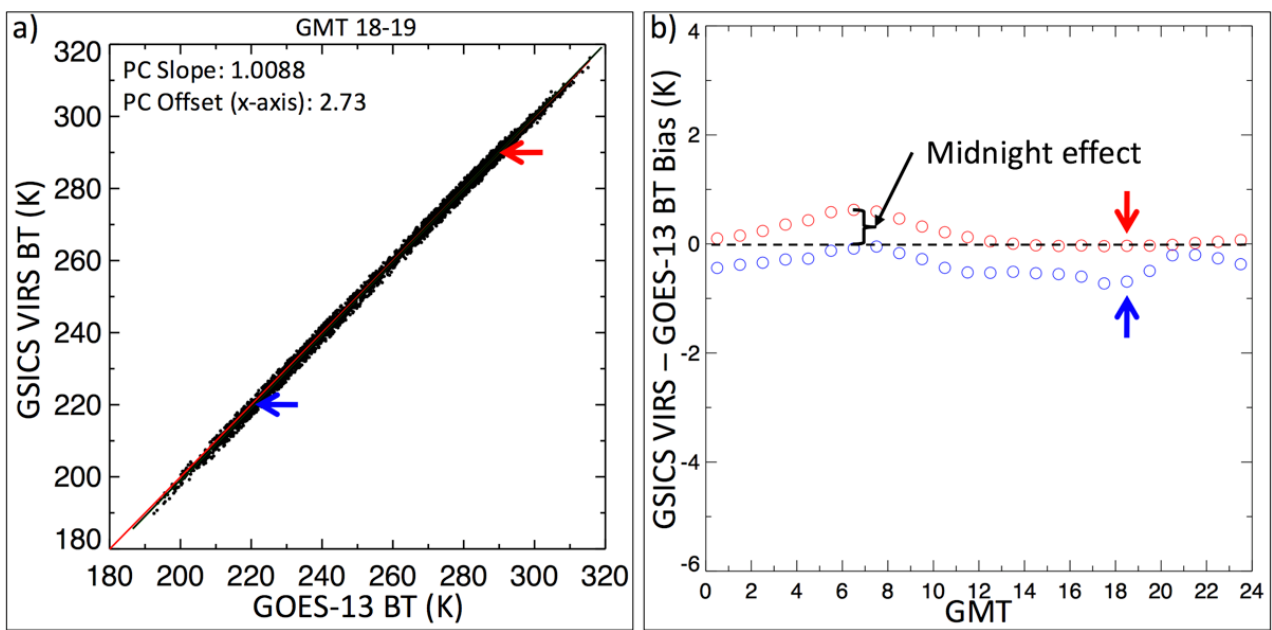

Figure 7. GOES-13 and VIRS (GSICS-referenced) 11- $\mu \mathrm{m}$ BT ray-matched bias results for a) 18-19 GMT culminated from May through December 2010 and January through April 2012 with principle components slope and x-axis offset. The blue and red arrows correspond to BT-dependent VIRS (GSICS-referenced) minus GOES-13 biases at 290 and $220 \mathrm{~K}$ as shown on the b) hourly bias plot. The magnitude of the midnight effect is illustrated (bracket). As such, the red arrow also indicates the minimum 290-K BT, occurring at 18 GMT (13 LT), which when subtracted from the maximum 290-K BT at 06 GMT (01 LT) quantifies the midnight effect.

A GSICS-referenced GOES-13 BT is attained by standardizing the original GOES-13 (observed) BT via GMTinterval-specific VIRS (GSICS-referenced) and GEOsat ray-matched slopes and offsets. This standardization is achieved as follows:

$$
B T_{\text {GEOsat(GSICS-referenced })}=\operatorname{slope}(G M T \text { interval }) \times\left(B T_{G E O s a t(o b s e r v e d)}-\text { off set }(G M T \text { interval })\right)
$$

Note that the reference GMT should always be based on the GEOsat sub-satellite longitude and not the imager scan location on the Earth's surface because the midnight effect is caused by the influence of excessive heat on the satellite itself and not by the Earth-reflected solar signal. That is, the midnight effect is independent of sun angle at the Earth's surface, but dependent on incident solar radiation upon the satellite instrument surfaces. Employing Eq. 2 for all GEOsats should facilitate inter-consistency among all concurrent imagers during the VIRS data record (1998 to 2012), provided that at least one year of GEOsat overlap is available. The hourly GEOsat BT corrections can then quantify the midnight effect for each GEOsat (see Section 3). A minimum of one full year of GEOsat and VIRS ray-matched BT pairs is required to successfully produce hourly GEOsat BT corrections. The GEOsat BT may have small seasonal dependencies, which are not removed using the technique highlighted in this manuscript. If time periods less than a year are utilized, the seasonal biases may alias into the diurnal corrections.

\subsection{Spectral Band Adjustment Factor}

Spectral differences between VIRS and the GEOsat imagers were accounted for during the hourly cross-calibration steps via convolution of footprint IASI hyper-spectral radiance measurements over the reference and target instrument spectral response functions (SRFs). The IASI footprint reference and target instrument pseudo radiance pairs are converted to BT via the Planck function and the SRF-convolved hyper-spectral IASI wavelength. The BT pseudo radiance pairs are regressed as a $2^{\text {nd }}$-degree polynomial, the coefficients of which constitute the spectral band adjustment factor (SBAF). Applying the SBAF to the reference sensor BT values yields the expected target sensor BT value, and thus SRF disparity is corrected for. The SBAFs were computed using NASA Langley Satellite Calibration online tools (https://satcorps.larc.nasa.gov). Further information regarding the tools and SBAF methodology/validation are found in Scarino et al. ${ }^{17}$.

Tables 1 and 2 list the $2^{\text {nd }}$-degree polynomial SBAF coefficients for the indicated reference and target pairings, for the 11- and 12- $\mu \mathrm{m}$ spectral channels, or bands (B), respectively. For the first step of normalizing VIRS to the Met-9GSICS standard, Met-9-GSICS is treated as the true reference to which the SBAF is applied in order to achieve consistency with the target sensor, VIRS. Using the now spectrally consistent Met-9 (GSICS-corrected) and VIRS crosscalibration coefficients, VIRS is adjusted to Met-9 (GSICS-corrected), and becomes the true reference to which an 
Table 1. Spectral band adjustment coefficients for the 11- $\mu \mathrm{m}$ reference and target satellite instrument band (B) pairings in terms of BT. Coefficient $A 2$ is the $2^{\text {nd }}$-degree non-linear term, $A 1$ is the $1^{\text {st }}$-degree linear term, and $A 0$ is the constant term. The imager channel numbers used in this study are given after the decimal in the first two columns.

\begin{tabular}{|c|c|c|c|c|}
\hline Reference.B (11 um) & Target.B (11 $\mu \mathrm{m})$ & A2 & A1 & AO \\
\hline TRMM-VIRS.4 & GOES-8.4 & $-3.1011 e-5$ & 1.0162 & -1.9914 \\
\hline TRMM-VIRS.4 & GOES-9.4 & $-2.6589 e-5$ & 1.0144 & -1.8329 \\
\hline TRMM-VIRS.4 & GOES-10.4 & $-5.4738 e-5$ & 1.0285 & -3.4988 \\
\hline TRMM-VIRS.4 & GOES-11.4 & $1.2930 \mathrm{e}-6$ & 1.0002 & -0.11499 \\
\hline TRMM-VIRS.4 & GOES-12.4 & $-1.2455 e-5$ & 1.0071 & -0.93831 \\
\hline TRMM-VIRS.4 & GOES-13.4 & $-6.5069 e-5$ & 1.0334 & -4.0579 \\
\hline TRMM-VIRS.4 & GOES-15.4 & $-3.8600 e-5$ & 1.0202 & -2.4833 \\
\hline TRMM-VIRS.4 & MTSAT-1.2 & $8.0014 \mathrm{e}-5$ & 0.96024 & 4.7074 \\
\hline TRMM-VIRS.4 & MTSAT-2.2 & $8.8172 e-5$ & 0.95683 & 5.0605 \\
\hline TRMM-VIRS.4 & GMS-5.2 & $8.9576 \mathrm{e}-5$ & 0.95457 & 5.4426 \\
\hline TRMM-VIRS.4 & Meteosat-5.2 & $5.4343 e-4$ & 0.71767 & 34.503 \\
\hline TRMM-VIRS.4 & Meteosat-7.2 & $6.3109 \mathrm{e}-4$ & 0.66919 & 40.691 \\
\hline TRMM-VIRS.4 & Meteosat-8.9 & $3.9015 e-5$ & 0.98082 & 2.2352 \\
\hline Meteosat-9.9 & TRMM-VIRS.4 & $-2.5682 e-5$ & 1.0127 & -1.4941 \\
\hline
\end{tabular}

Table 2. Spectral band adjustment coefficients for the $12-\mu \mathrm{m}$ reference and target satellite instrument band (B) pairings in terms of BT. Coefficient $A 2$ is the $2^{\text {nd }}$-degree non-linear term, $A 1$ is the $1^{\text {st }}$-degree linear term, and $A O$ is the constant term.

\begin{tabular}{|l|l|r|r|r|}
\hline Reference. B $(12 \mu \mathrm{m})$ & Target.B $(12 \mu \mathrm{m})$ & \multicolumn{1}{|c|}{ A2 } & \multicolumn{1}{|c|}{ A1 } \\
\hline TRMM-VIRS.5 & GOES-8.5 & $3.6641 \mathrm{e}-5$ & 0.98264 & 2.0230 \\
\hline TRMM-VIRS.5 & GOES-9.5 & $4.8406 \mathrm{e}-5$ & 0.97531 & 3.0306 \\
\hline TRMM-VIRS.5 & GOES-10.5 & $5.0325 \mathrm{e}-5$ & 0.97074 & 3.8883 \\
\hline TRMM-VIRS.5 & GOES-11.5 & $4.7599 \mathrm{e}-5$ & 0.97478 & 3.1745 \\
\hline TRMM-VIRS.5 & MTSAT-1.3 & $6.0929 \mathrm{e}-5$ & 0.97079 & 3.4255 \\
\hline TRMM-VIRS.5 & MTSAT-2.3 & $4.4101 \mathrm{e}-5$ & 0.98018 & 2.2087 \\
\hline TRMM-VIRS.5 & GMS-5.3 & $-2.1308 \mathrm{e}-4$ & 1.1209 & -15.750 \\
\hline TRMM-VIRS.5 & Meteosat-8.10 & $2.4655 \mathrm{e}-5$ & 0.99134 & 0.74054 \\
\hline Meteosat-9.10 & TRMM-VIRS.5 & $-3.7751 \mathrm{e}-5$ & 1.0178 & -2.0678 \\
\hline
\end{tabular}

SBAF is applied in order to be spectrally consistent with each GEOsat target. The SBAFs were determined from IASI footprints taken over the tropical western pacific in order to maximize the dynamic range of the measurements, rather than over each respective GEOsat domain. That is, with consistent observation of the clear-sky ocean and deep convective clouds from four seasonal months, sampling should be sufficient on both the cold and warm ends of the BT range, and thus the SBAF can appropriately account for temperature-induced spectral biases.

\section{RESULTS AND DISCUSSION}

Table 3 shows the hour, based on the local time of the GEOsat longitude, of the maximum and minimum 11- $\mu \mathrm{m}$ BT biases, along with the BT bias difference between the maximum and minimum, for Met-9-GSICS-consistent VIRS, i.e., VIRS (GSICS-referenced), and the indicated GEOsat at a GEOsat reference temperature of $290 \mathrm{~K}$. For example, the maximum BT bias at $290 \mathrm{~K}$ for GOES-13 in Fig. 7b (red circles) occurs near 01:00 LT, the minimum bias occurs 12 hours later near 13:00 LT, and the difference is $0.65 \mathrm{~K}$. Similarly shown, in the penultimate row, are the local time and 
Table 3. Local time of the maximum and minimum 11- $\mu \mathrm{m}$ 290-K BT bias between VIRS (GSICS-referenced) and a GEOsat, and the BT (maximum - minimum) bias difference. Bold rows indicate three-axis-stabilized platforms whereas the remaining are spin-stabilized platforms. Respective average results are shown in $10^{\text {th }}$ row and the final row.

\begin{tabular}{|l|r|r|r|}
\hline $\begin{array}{l}\text { Satellite(Reference) } \\
11 \mu m\end{array}$ & $\begin{array}{l}\text { Local Time of } \\
\text { BT Bias } \\
\text { Maximum } \\
\text { (HH:MM) }\end{array}$ & $\begin{array}{l}\text { Local Time of } \\
\text { BT Bias } \\
\text { Minimum } \\
\text { (HH:MM) }\end{array}$ & \multicolumn{2}{l}{$\begin{array}{l}\text { BT Bias } \\
\text { Difference } \\
\text { (K) }\end{array}$} \\
\hline GOES-8(VIRS-Met-9-GSICS) & $\mathbf{0 1 : 0 0}$ & $\mathbf{1 0 : 0 0}$ & $\mathbf{0 . 6 1}$ \\
\hline GOES-9(VIRS-Met-9-GSICS) & $\mathbf{0 1 : 0 0}$ & $\mathbf{0 9 : 0 0}$ & $\mathbf{0 . 4 9}$ \\
\hline GOES-10(VIRS-Met-9-GSICS) & $\mathbf{0 3 : 0 0}$ & $\mathbf{1 3 : 0 0}$ & $\mathbf{0 . 4 9}$ \\
\hline GOES-11(VIRS-Met-9-GSICS) & $\mathbf{0 1 : 0 0}$ & $\mathbf{0 9 : 0 0}$ & $\mathbf{0 . 5 6}$ \\
\hline GOES-12(VIRS-Met-9-GSICS) & $\mathbf{0 1 : 0 0}$ & $\mathbf{1 1 : 0 0}$ & $\mathbf{0 . 5 6}$ \\
\hline GOES-13(VIRS-Met-9-GSICS) & $\mathbf{0 1 : 0 0}$ & $\mathbf{1 3 : 0 0}$ & $\mathbf{0 . 6 5}$ \\
\hline GOES-15(VIRS-Met-9-GSICS) & $\mathbf{0 1 : 0 0}$ & $\mathbf{1 0 : 0 0}$ & $\mathbf{0 . 3 3}$ \\
\hline MTSAT-1R(VIRS-Met-9-GSICS) & $\mathbf{0 1 : 0 0}$ & $\mathbf{1 0 : 0 0}$ & $\mathbf{0 . 4 4}$ \\
\hline MTSAT-2(VIRS-Met-9-GSICS) & $\mathbf{0 3 : 0 0}$ & $\mathbf{0 9}: 00$ & $\mathbf{0 . 2 3}$ \\
\hline All Three-axis-stabilized & $\mathbf{0 1 : 2 6} \pm \mathbf{0 0 : 5 3}$ & $\mathbf{1 0 : 2 6} \pm \mathbf{0 1 : 3 5}$ & $\mathbf{0 . 4 8} \pm \mathbf{0 . 1 3}$ \\
\hline GMS-5(VIRS-Met-9-GSICS) & $19: 00$ & $09: 00$ & 0.16 \\
\hline Met-5(VIRS-Met-9-GSICS) & $12: 00$ & $03: 00$ & 0.21 \\
\hline Met-7(VIRS-Met-9-GSICS) [0 ${ }^{\circ}$ E] & $12: 00$ & $05: 00$ & 0.42 \\
\hline Met-7(VIRS-Met-9-GSICS) [57 ${ }^{\circ}$ E] & $14: 00$ & $05: 00$ & 0.36 \\
\hline Met-8(VIRS-Met-9-GSICS) & $10: 00$ & $23: 00$ & 0.10 \\
\hline VIRS(Met-9-GSICS) & $22: 00$ & $09: 00$ & 0.10 \\
\hline All Spin-stabilized & $14: 50 \pm 04: 40$ & $05: 00 \pm 03: 47$ & $0.22 \pm 0.14$ \\
\hline
\end{tabular}

BT difference of the maximum and minimum BT biases for Met-9 (GSICS-corrected) with respect to a VIRS (GSICSreferenced) BT of $290 \mathrm{~K}$. The rows in bold lettering indicate satellite platforms that utilize three-axis stabilization, whereas the non-bolded rows indicate satellite platforms that utilize spin stabilization. The average and standard deviation uncertainty of each column follows each respective stabilization method series.

For three-axis-stabilized satellites, the time of maximum BT bias occurs in the hours immediately following local midnight. In most cases, 01:00 LT is the hour of maximum bias, with the average occurring near 01:26 LT, and with a standard deviation of 53 minutes. This timing is consistent with the midnight effect ${ }^{6,7,8,11}$. The minimum BT bias timing is less consistent, and occurs during local morning to early-afternoon hours, with an average of 10:26 LT and uncertainty of 95 minutes. The difference in BT bias between these times extends from 0.23 to $0.65 \mathrm{~K}$, with an average of $0.48 \pm 0.13 \mathrm{~K}$. The lower standard deviation for the timing of the maximum bias compared to that of the minimum bias is indicative of the systematic occurrence of the anomalous IR-imager BT drift near local midnight. That is, the local maxima in the red circle series of Fig. $7 \mathrm{~b}$ is consistent across the three-axis stabilized platforms with respect to local time. The local minimum, though less consistent in timing, occurs at least 6 hours later (up to 12 hours with an average of 9 hours), which is likely consistent with the time necessary for the heated instrument surfaces to equilibrate.

For spin-stabilized satellites, maximum and minimum timing consistency is much more disparate. The average occurrence for the maximum bias is 14:50 LT with a 280-minute uncertainty. Minimum bias timing has a slightly smaller, but considerable, standard deviation of 227 minutes at 05:00 LT. The respective averages indicate a pattern that is quasi-consistent with time latencies for maximum surface solar heating and radiative cooling, but that arrangement seems to be primarily driven by the Met- 5 and Met- 7 cases. When considering only Met- 5 and Met-7 (for all three longitudinal three positions), the average timing for the maximum bias is 12:40 LT with a standard deviation of 69 minutes, and the average timing for the minimum bias is 04:20 LT, also with a standard deviation of 69 minutes. For the 
remaining spin-stabilized satellites, the average timing for the maximum bias is 17:00 LT with an uncertainty of 374 minutes, and the average timing for the minimum bias is 05:40 LT with an uncertainty of 346 minutes. Thus the Met-5 and Met-7 satellites appear to have a diurnal bias dependency tied to surface solar heating and radiative cooling, which is distinct from the midnight effect. Thus it is unsurprising that of the $0.22 \pm 0.14-\mathrm{K}$ average bias (which is still less than half of that for the three-stabilized satellites), the contribution from Met-5 and Met-7 is $0.33 \pm 0.11 \mathrm{~K}$, and the contribution from the remaining spin-stabilized satellites is $0.12 \pm 0.03 \mathrm{~K}$.

The GMS-5, Met-8, and Met-9 satellites therefore demonstrate no meaningful diurnal dependency given their significantly large local maximum/minimum bias timing uncertainties and small bias difference. The older, firstgeneration Met-5 and Met-7 cases also indicate no subjection to the midnight effect, which is in line with expectations for spin-stabilized satellites. Although these broad-SRF Meteosat imagers appear to be influenced by another source of diurnal bias, perhaps driven by surface radiative flux, this dependency matters little because it is inherently accounted for by the VIRS (GSICS-referenced) adjustment.

Table 4 shows the same platform series listings of maximum/minimum bias timings and differences as Table 3, but for the 12- $\mu \mathrm{m}$ spectral bands. The GOES-12, GOES-13, and GOES-15 satellites are absent from this analysis given that their $12-\mu \mathrm{m}$ channel was replaced with a $13.3-\mu \mathrm{m}$ channel. Although the influence of the midnight effect is less pronounced for $12-\mu \mathrm{m}$ wavelengths ${ }^{6,7,8,11}$, the systematic timing of maximum BT biases is still apparent for the three-axis stabilized satellites. Timing for the maximum bias is consistently near midnight, with an average of 00:40 and standard deviation of 62 minutes (with consideration of midnight transition). Timing for the minimum bias following the maximum bias is much less reliable than that for the 11- $\mu \mathrm{m}$ case, however, being roughly 8-9 hours later and occurring on average at 09:20 with an uncertainty of 245 minutes. This high uncertainty in minimum bias timing may suggest a weaker systematic dependency for thermal equilibrium lag following midnight effect heating than that for the $11-\mu \mathrm{m}$ channel, perhaps due to the weaker influence of the anomaly at $12 \mu \mathrm{m}$.

The Table 4 local maximum timing for the spinning satellites is expectedly inconsistent, as in Table 3, with a standard deviation in excess of 240 minutes. As before, such inconsistency demonstrates the non-influence of the midnight effect for spin-stabilized platforms. The mean bias difference and uncertainty results are comparable to that for the same instruments at the $11-\mu \mathrm{m}$ wavelengths, with values of $0.17 \pm 0.10 \mathrm{~K}$. The spin-stabilized satellite mean BT bias result is less than half of that for the three-axis-stabilized satellites. Therefore, similar to the 11- $\mu \mathrm{m}$ analysis, these results indicate the susceptibility of three-axis stabilized satellites to heat stress, which has a systematic effect on measured BT, but which can be accounted for with these hourly VIRS (GSICS-referenced) consistency corrections.

Table 4. Local time of the maximum and minimum 12- $\mu \mathrm{m}$ 290-K BT bias between VIRS (GSICS-referenced) and a GEOsat, and the BT (maximum - minimum) bias difference. Bold rows indicate three-axis-stabilized platforms whereas the remaining are spin-stabilized platforms. Respective average results are shown in $7^{\text {th }}$ row and the final row.

\begin{tabular}{|c|c|c|c|}
\hline $\begin{array}{l}\text { Satellite(Reference) } \\
12 \mu \mathrm{m}\end{array}$ & $\begin{array}{l}\text { Local Time of } \\
\text { BT Bias } \\
\text { Maximum } \\
\text { (HH:MM) }\end{array}$ & $\begin{array}{l}\text { Local Time of } \\
\text { BT Bias } \\
\text { Minimum } \\
\text { (HH:MM) }\end{array}$ & $\begin{array}{l}\text { BT Bias } \\
\text { Difference } \\
\text { (K) }\end{array}$ \\
\hline GOES-8(VIRS-Met-9-GSICS) & 01:00 & 09:00 & 0.47 \\
\hline GOES-9(VIRS-Met-9-GSICS) & 01:00 & 06:00 & 0.26 \\
\hline GOES-10(VIRS-Met-9-GSICS) & 02:00 & 10:00 & 0.20 \\
\hline GOES-11(VIRS-Met-9-GSICS) & 23:00 & 08:00 & 0.32 \\
\hline MTSAT-1R(VIRS-Met-9-GSICS) & 01:00 & $17: 00$ & 0.37 \\
\hline MTSAT-2(VIRS-Met-9-GSICS) & 00:00 & 06:00 & 0.61 \\
\hline All Three-axis-stabilized & $00: 40 \pm 01: 02$ & 09:20 $\pm 04: 05$ & $0.37 \pm 0.15$ \\
\hline GMS-5(VIRS-Met-9-GSICS) & $17: 00$ & 08:00 & 0.16 \\
\hline Met-8(VIRS-Met-9-GSICS) & 09:00 & $13: 00$ & 0.07 \\
\hline VIRS(Met-9-GSICS) & $12: 00$ & 05:00 & 0.27 \\
\hline All Spin-stabilized & $12: 40 \pm 04: 02$ & 08:40 $\pm 04: 02$ & $0.17 \pm 0.10$ \\
\hline
\end{tabular}



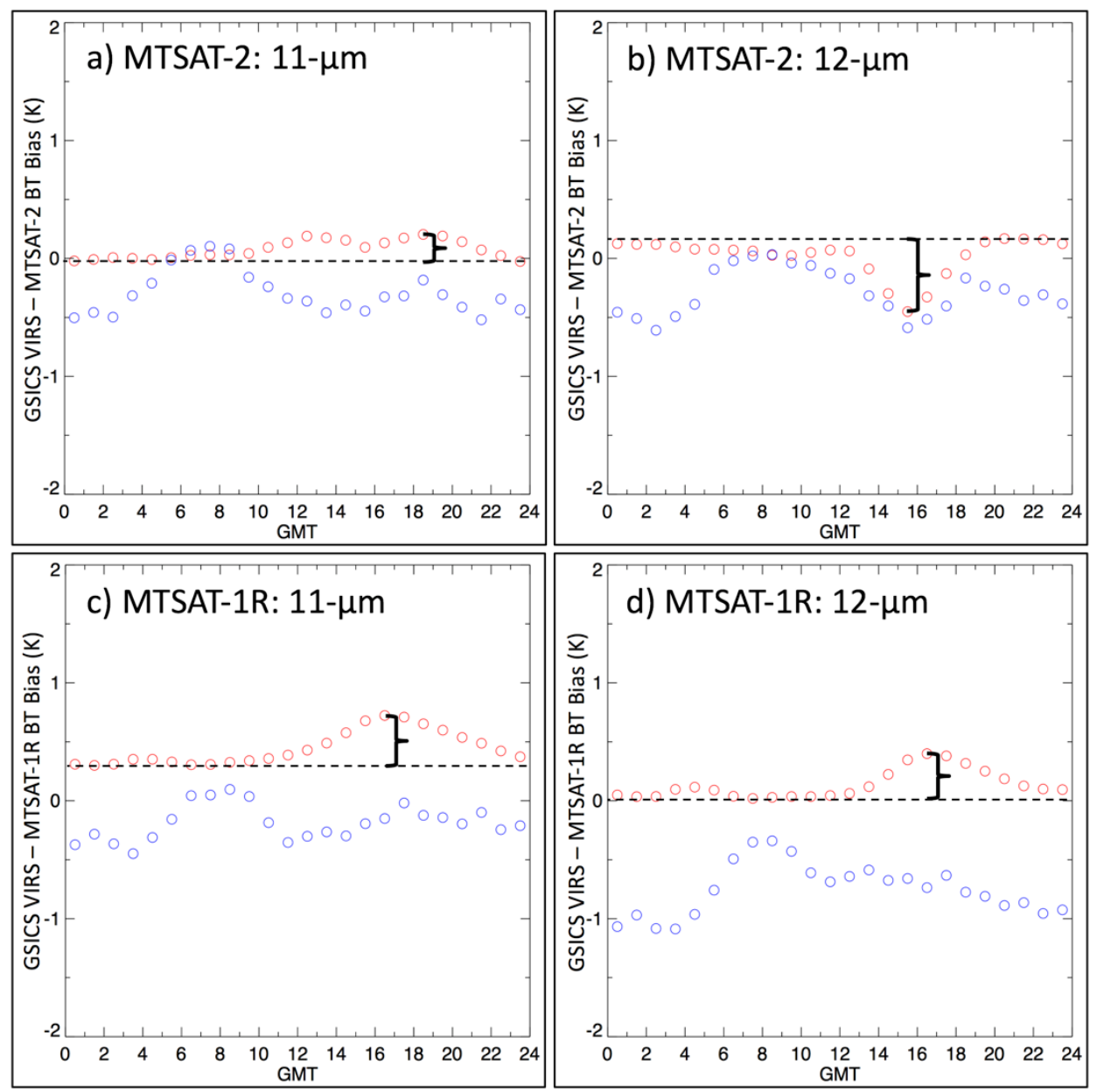

Figure 8. a) VIRS (GSICS-referenced) minus MTSAT-2 hourly $11-\mu \mathrm{m}$ BT biases at $290 \mathrm{~K}$ (red circles) and $220 \mathrm{~K}$ (blue circles). The amplitude of the 290-K diurnal BT bias (bracket) is also shown as described in Fig. 7b. b) Same as a) except for the 12- $\mu \mathrm{m}$ channel. c) VIRS (GSICS-referenced) minus MTSAT-1R hourly 11- $\mu \mathrm{m}$ BT biases at 290 and $220 \mathrm{~K}$. d) Same as c) except for the $12-\mu \mathrm{m}$ channel. The MTSAT-2 $\left(145^{\circ} \mathrm{E}\right)$ and MTSAT-1R (140 $\left.{ }^{\circ} \mathrm{E}\right)$ satellites are located in a similar spatial position in terms of local solar influence. The MTSAT-1R midnight (16 GMT) effect is typical of other three-axis stabilized satellites.

The MTSAT-2 12- $\mu \mathrm{m}$ channel was found to have an unusual midnight effect - seemingly an opposite effect compared compared to that of the corresponding 11- $\mu \mathrm{m}$ channel (Fig. 8) and all other three-axis-stabilized satellites. That is, the heat stress from the midnight anomaly induces a warm bias in MTSAT-2 measurements, rather than the typical cool bias, therefore the maximum/minimum timings in Table 4 are reversed with respect to the other satellites, but accurate for the purposes of this manuscript in quantifying the magnitude of BT bias mitigation afforded by the calibration reference transfer through VIRS. The exact cause is unknown, but perhaps the midnight effect mitigation was overcompensated.

\section{CONCLUSIONS}

A VIRS-based GEOsat IR imager diurnal calibration method was presented, which has the potential to quantify and correct for the midnight BT drift anomaly that is common among three-axis stabilized GEOsat IR imagers. The VIRS instrument is ideal for this task given that the precessing orbit of TRMM allows for full diurnal resolution every 46 days. The method first involves establishing the long-term stability of the VIRS IR imager relative to the well-calibrated Aqua-MODIS IR bands. Next, VIRS is normalized hourly using Met-9 GEOsat IR imager ray-matched comparisons. The Met-9 satellite is spin-stabilized and therefore is not susceptible to this specific midnight BT anomaly. The Met-9 
BT were first corrected using the GSICS calibration coefficients based on the IASI calibration reference. The VIRS (GSICS-referenced) BT were found to be consistent relative to direct VIRS (observed) and IASI BT comparisons at the IASI overpass times. The VIRS (GSICS-referenced) data are matched with concurrent GEOsat imagers to yield hourly calibration coefficients for achieving BT uniformity across GEOsat imagers, which are referenced to the GSICS calibration source. The calibration adjustment results indicate a systematic maximum in BT bias correction occurring shortly after local midnight for all three-axis-stabilized GEOsat imagers, for both the 11- and 12- $\mu \mathrm{m}$ channels. No such occurrence is notable for the spin-stabilized satellite instruments. The range in BT bias correction is also greater for the three-axis-stabilized platforms than that for spin-stabilized. These results are expected given the nature of the heat stress midnight anomaly. Regardless of the need to quantify and correct for a midnight effect, this VIRS-based calibration method is effective for transferring the IASI-based GSICS calibration reference to all concurrent IR imagers with no diurnal dependency.

\section{ACKNOWLEDGMENTS}

This work was supported by the NASA Research Opportunities in Space and Earth Sciences for Satellite Calibration Interconsistency Studies and the NASA Clouds and the Earth's Radiant Energy System (CERES) project.

\section{REFERENCES}

[1] Bedka, K. M. and K. Khlopenkov, "A probabilistic multispectral pattern recognition method for detection of overshooting cloud tops using passive satellite imager observations," J. Appl. Meteor. And Climatol., In press (2016).

[2] Doelling, D., N. Loeb, D. Keyes, M. Nordeen, D. Morstad, C. Nguyen, B. Wielicki, D. Young, and M. Sun, 2013, "Geostationary enhanced temporal interpolation for CERES flux products," J. Atmos. Oceanic Technol. Vol. 30, No. 6, June 2013: 1072-1090, doi:10.1175/JTECH-D-12- 00136.1

[3] Doelling, D.R., C.O. Haney, B.R. Scarino, A. Gopalan, and R. Bhatt, 2016, "Improvements to the geostationary visible imager ray-matching calibration algorithm for CERES edition 4," J. Atmos. Oceanic Technol., Vol. 33 No. 12, pp. 2679-2698, DOI: 10.1175/JTECH-D-16-0113.1

[4] Bhatt, R., D. R. Doelling, D. L. Morstad, B. R. Scarino, and A. Gopalan, "Desert-based absolute calibration of successive geostationary visible sensors using a daily exoatmospheric radiance model," IEEE Transactions on Geoscience and Remote Sensing, 51, 3670-3682 (2013).

[5] Goldberg, M., G. Ohring, J. Butler, C. Cao, R. Datla, D. Doelling, V. Gärtner, T. Hewison, B. Iacovazzi, D. Kim, T. Kurino, J. Lafeuille, P. Minnis, D. Renaut, J. Schmetz, D. Tobin, L. Wang, F. Weng, X. Wu, F. Yu, P. Zhang, and T. Zhu, "The Global Space-based Inter-Calibration System (GSICS)," Bull. Amer. Meteor. Soc. 92(4), 467-475 (2011).

[6] Hilton, F. et al., "Hyperspectral earth observations from IASI: four years of accomplishments," Bull. Amer. Meteor. Soc., doi: 10.1175/BAMS-D-11-00027.1 (2011).

[7] Johnson, R. X. and M. P. Weinreb, "GOES-8 imager midnight effects and slope correction," Proc. SPIE, vol. 2812, pp. 596-607 (1996).

[8] Weinreb, M. and D. Han, "Correction for GOES imager midnight calibration errors," NOAA Office of Satellite and Product Operations, (2003).

[9] Weinreb, M. and D. Han, "Implementation of midnight blackbody calibration correction (MBCC)," NOAA Office of Satellite and Product Operations, (2003).

[10] Wu, X., "NOAA-KMA collaboration on imager midnight IR calibration anomaly," GSICS WG Meeting, Madison, WI, 22 Mar 2017.

[11] Hillger, D. W., T. J. Schmit, et al., "The GOES-15 science test: imager and sounder radiance and product validations," U.S. Department of Commerce National Oceanic and Atmospheric Administration National Environmental Satellite, Data, and Information Service, NOAA Technical Report NESDIS 141 (2011).

[12] Yu, F., X. Wu, M. K. Rama Varma Raja, Y. Li, L. Wang, and M. Goldberg, "Diurnal and scan angle variations in the calibration of GOES imager infrared channels," IEEE Trans. Geosci. Remote Sens., vol. 51, no. 1, pp. 671-683 (2013). 
[13] Mittaz, J. and A. Harris, "Using AATSR as radiance calibration reference for NOAA satellites," in Proc. 2nd MERIS/(A)ASTR User Workshop, Frascati, Italy, Sep. 2008.

[14] Gunshor, M., T. Schmit, P. Menzel, and D. Tobin, "Intercalibration of broadband geostationary imagers using AIRS,” J. Atmos. Ocean. Technol., vol. 26, no. 4, pp. 746-758, Apr. 2009.

[15] Rama Varma Raja, M. K., X. Wu, F. Yu, and L. Wang, "Assessment of mid-night blackbody calibration correction (MBCC) using the global space-based inter-calibration system (GSICS)," in Proc. SPIE, 2009, vol. 7456, p. 745 603.

[16] Xiong, X., K.-F. Chiang, A. Wu, W. L. Barnes, B. Guenther, and V. V. Salomonson, "Multiyear on-orbit calibration and performance of Terra MODIS thermal emissive bands," in IEEE Trans. Geosci. Remote Sens., vol. 46, no. 6, pp. 1790-1803 (2008).

[17] Scarino, B. R., D. R. Doelling, P. Minnis, A. Gopalan, T. Chee, R. Bhatt, C. Lukashin, and C. O. Haney, "A webbased tool for calculating spectral band difference adjustment factors derived from SCIAMACHY hyperspectral data," IEEE Trans. Geosci. Remote Sens., vol. 54, no. 5, pp. 2529-2542 (2016). 